\title{
't Hooft loops and perturbation theory
}

\section{Philippe de Forcrand*}

Institute for Theoretical Physics, ETH Zürich, CH-8093 Zürich, Switzerland CERN, Physics Department, TH Unit. CH-1211 Genève 23, Switzerland

E-mail: forcrandephys.ethz.ch

\section{Biagio Lucini}

Institute for Theoretical Physics, ETH Zürich, CH-8093 Zürich, Switzerland

E-mail: luciniephys.ethz.ch

\section{David Noth}

Paul Scherrer Institut, CH-5232 Villigen PSI, Switzerland

E-mail: nothd@phys.ethz.ch

We show that high-temperature perturbation theory describes extremely well the area law of $S U(N)$ spatial 't Hooft loops, or equivalently the tension of the interface between different $Z_{N}$ vacua in the deconfined phase. For $S U(2)$, the disagreement between Monte Carlo data and lattice perturbation theory for $\tilde{\sigma}(T) / T^{2}$ is less than $2 \%$, down to temperatures $\mathscr{O}(10) T_{c}$. For $S U(N), N>3$, the ratios of interface tensions, $\left(\tilde{\sigma}_{k} / \tilde{\sigma}_{1}\right)(T)$, agree with perturbation theory, which predicts tiny deviations from the ratio of Casimirs, down to nearly $T_{c}$. In contrast, individual tensions differ markedly from the perturbative expression. In all cases, the required precision Monte Carlo measurements are made possible by a simple but powerful modification of the 'snake' algorithm.

XXIIIrd International Symposium on Lattice Field Theory

25-30 July 2005

Trinity College, Dublin, Ireland

\footnotetext{
*Speaker.
} 


\section{Introduction}

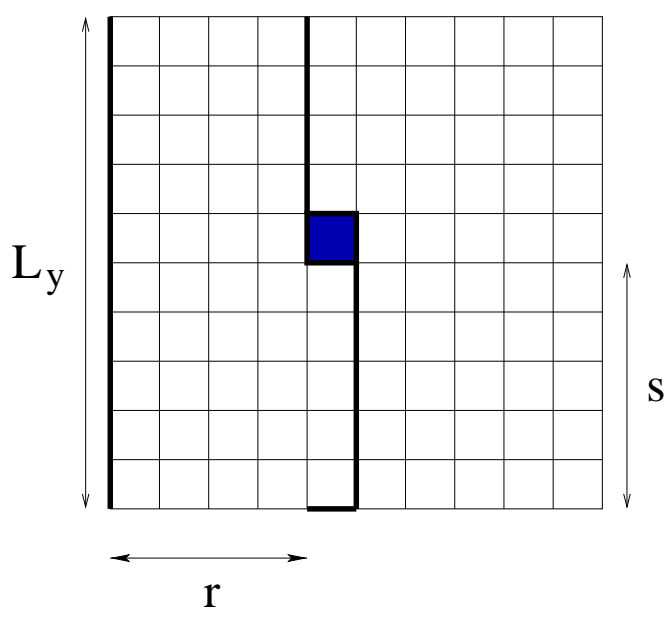

Figure 1: The interface tension is extracted from the measured ratio $\exp \left(-\widetilde{\sigma} a^{2}\right)$ of partition functions of two systems with partial interfaces, which differ by the single shaded plaquette.

't Hooft has shown that, in Yang-Mills theories at zero temperature, and in the absence of massless modes, an area law for the Wilson loop implies a perimeter law for the 't Hooft loop, and vice versa. This dual behaviour carries over at fi nite temperature [1]: while the temporal Wilson loop adopts a perimeter law in the deconfi ned phase $T>T_{c}$, the spatial 't Hooft loop acquires an area law.

This can be shown without any simulations. A spatial 't Hooft loop $\tilde{W}(\partial \tilde{\Sigma})$ bounding a surface $\tilde{\Sigma}$, say, in the $(x, y)$ plane, is created on the lattice by "flipping" a stack of $(z, t)$ plaquettes, one per plane pierced by $\tilde{\Sigma}$. "Flipping" means that the plaquette matrix $U_{P}$ is multiplied by a center element $z_{k}=\exp \left(i \frac{2 \pi k}{N}\right) \mathbf{1}$ before its trace is evaluated. The corresponding partition function $Z_{\text {flipped }}$ gives the 't Hooft loop expectation value via $\langle\tilde{W}(\tilde{\Sigma})\rangle=Z_{\text {flipped }} / Z_{\mathrm{pbc}}$, where the denominator corresponds to the usual action, with periodic boundary conditions.

It is easy to move the stack of plaquettes to a corner of the $(z, t)$ plane, then absorb the phase factor $z_{k}$ in the boundary condition of the temporal link $U_{t}$ :

$$
U_{t}\left(z+L_{z}, t=t_{0}\right)=z_{k} U_{t}\left(z, t=t_{0}\right)
$$

$Z_{\text {flipped }}$ then becomes the usual partition function (no flipped plaquettes) of a system where a twist has been enforced on the Polyakov loop $P$ :

$$
P\left(x, y, z+L_{z}\right)=z_{k} P(x, y, z) \text { for } x, y \in \tilde{\Sigma}
$$

When $T>T_{c}$, the center symmetry is broken, and the twist above causes an interface to appear, perpendicular to the $z$-direction, because the Polyakov loop lies in different $Z_{N}$ sectors at $z=0$ and $z=L_{z}$. The associated increase in free energy can be ascribed to the interface tension, or equivalently to the 't Hooft loop [dual] string tension $\widetilde{\sigma}$. These are two names for the same observable.

This equivalence allows us to compare numerical results for $\widetilde{\sigma}$ with old perturbative calculations of the interface tension [2]. It also suggests a simple way to measure $\widetilde{\sigma}$, further simplifying the "snake" algorithm [3]: just increase the interface area by one plaquette, and measure the change in free energy $\exp \left(-\widetilde{\sigma} a^{2}\right)$ (see Fig. 1). We perform such simulations and comparisons for $S U(N)$, discussing fi rst the case $N=2[4]$ and then $N \geq 3$.

\section{2. $\mathrm{SU}(2)$}

We have repeated the 1-loop perturbative calculation of the interface tension of [2], on a lattice of $N_{t}$ time-slices. In fact, it amounts to substituting $p \rightarrow \hat{p}=\frac{2}{a} \sin \frac{p a}{2}$ in the fluctuation determinant. 


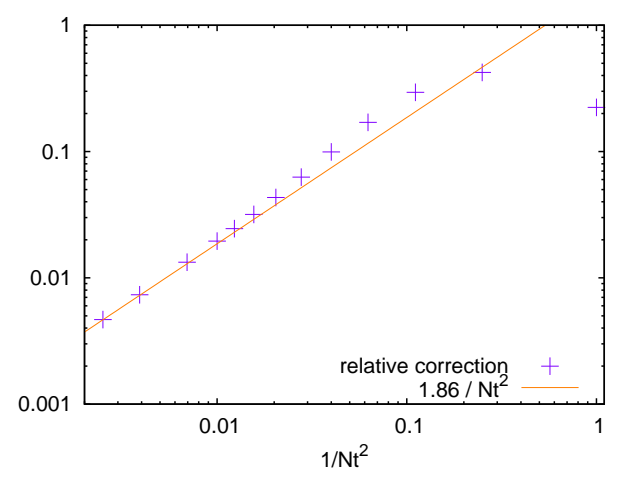

Figure 2: The relative correction $\left(C_{\text {lat }}\left(N_{t}\right)-1\right)$ to the interface tension $\widetilde{\sigma}$, for different temporal lattice sizes $N_{t}$.

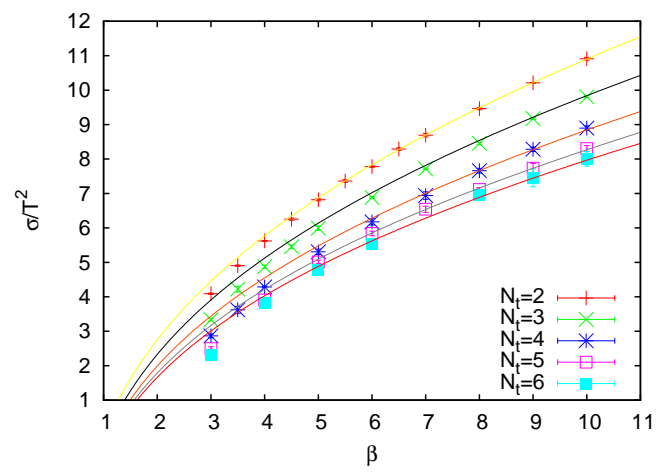

Figure 3: The dimensionless ratio $\widetilde{\sigma} / T^{2}\left(\beta, N_{t}\right)$ agrees excellently with the parameter-free perturbative formula eq.(2.2).

The resulting interface tension is multiplied by a coeffi cient $G_{\text {at }}\left(N_{t}\right)$, whose difference with 1 is shown in Fig. 2. The expected $1 / N_{t}^{2}$ behaviour does not set in until $N_{t} \gtrsim 10$, and for practical values of $N_{t}, C_{\text {lat }}\left(N_{t}\right)$ is large and not even monotonic. Thus, it provides an essential correction when computing $\widetilde{\sigma}$ on the lattice. The corresponding $S U(N)$ leading-order perturbative prediction is

$$
\frac{\tilde{\sigma}_{k}}{T^{2}}(T)=C_{\text {lat }}\left(N_{t}\right) \times \frac{4 \pi^{2}}{3 \sqrt{3}} \times \frac{1}{\sqrt{\left(g^{2} N\right)(T)}} \times k(N-k)
$$

Using the "snake"-like algorithm above, we have measured $\widetilde{\sigma}$ in $S U(2)$ for a wide range of couplings $\beta$ and lattice sizes $N_{t}$. As shown Fig. 3, excellent agreement is found with perturbation theory at $\mathscr{O}\left(g^{2}\right)$ [2], after converting the coupling from the improved scheme [5] to the lattice for $S U(2)$ :

$$
\frac{\tilde{\sigma}}{T^{2}}\left(\beta, N_{t}\right)=C_{\text {lat }}\left(N_{t}\right) \times \frac{2 \pi^{2}}{3 \sqrt{6}} \times \sqrt{\beta} \times\left(1-\left(0.21467+0.04644 \log N_{t}\right) \frac{4}{\beta}\right)
$$

The unknown $\mathscr{O}\left(g^{2}\right)$ contribution to $C_{\text {lat }}\left(N_{t}\right)$ appears to be very small. We can then convert $\left(\beta, N_{t}\right)$ to temperatures $T / \Lambda_{\overline{M S}}$, using 2-loop perturbation theory and $T_{c} / \Lambda_{\overline{M S}}=1.31$ (8) [6]. The data obtained at various lattice spacings collapse nicely, and the ratio of the measured over the perturbative $\widetilde{\sigma}$ remains within $\sim 2 \%$ of 1 , down to $\mathscr{O}(10) T_{c}$ where the measured $\widetilde{\sigma}$ decreases, since it has to vanish at the second-order transition $T=T_{c}$. See Fig. 4.

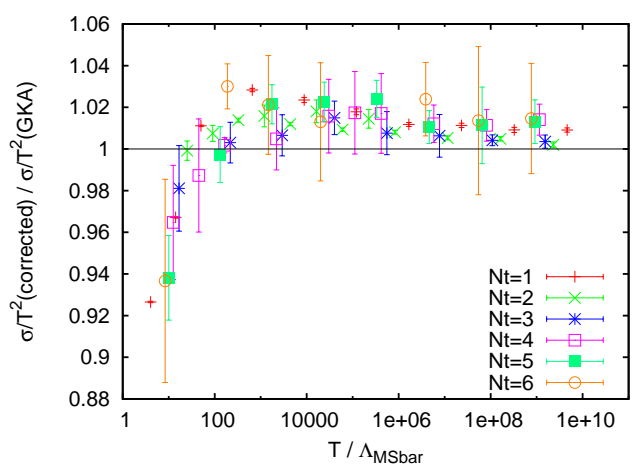

Figure 4: The ratio of the measured over the perturbative interface tension, as a function of temperature. Deviations are $\lesssim 2 \%$, down to the vicinity of $T_{c}$ where the measured $\widetilde{\sigma}$ must vanish. 

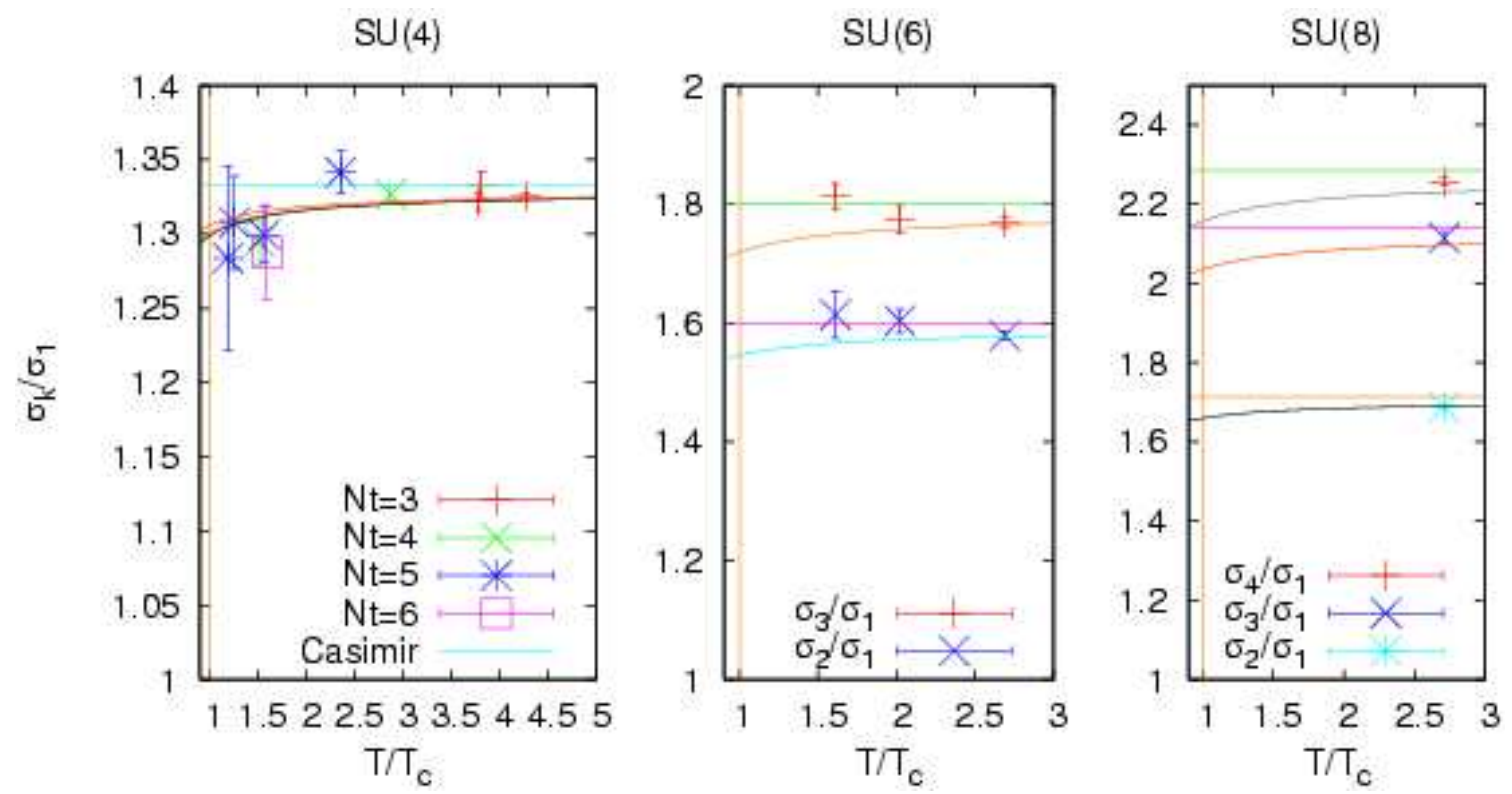

Figure 5: Ratios of interface tensions $\widetilde{\sigma}_{k} / \widetilde{\sigma}_{1}$, as a function of temperature, for $S U(4)$ (left), SU(6) (middle) and $S U(8)$ (right). The horizontal lines mark the Casimir values $\frac{k(N-k)}{N-1}$. The curves show the $\mathscr{O}\left(g^{3}\right)$ perturbative prediction of [7]. The 2 curves for $S U(4)$ correspond to $T_{c} / \Lambda_{\overline{M S}}=1.10$ and 1.35 .

\section{3. $\mathrm{SU}(\mathbf{N})$}

The same numerical method can be used for $S U(N)$ gauge theories. The interesting difference is that the $N$-fold degeneracy of the vacuum now allows for inequivalent interfaces. In the vacuum, the Polyakov loop can take values $z_{k}=\exp \left(i 2 \pi \frac{k}{N}\right) \mathbf{1} \in Z_{N}$. Interfaces separating two vacua $k_{1}, k_{2}$ rotate the Polyakov loop by $z_{k_{1}} z_{k_{2}}^{-1}=\exp \left(i 2 \pi \frac{k_{1}-k_{2}}{N}\right)$. The corresponding interface tension is $\widetilde{\sigma}_{k}$, with $k=k_{1}-k_{2} \bmod N$. Charge conjugation imposes $\widetilde{\sigma}_{k}=\widetilde{\sigma}_{N-k}$. This leaves $\operatorname{int}\left(\frac{N}{2}\right)$ independent interface tensions.

We measure them independently using the same setup of Fig. 1, where the "twisted" plaquette has action $\operatorname{Re} \operatorname{Tr} z_{k} U_{P}$. The temperature can be varied by changing the inverse coupling $\beta$ or the number $N_{t}$ of time-slices. To stay clear of a bulk fi rst-order transition for $N>3$, we chose $N_{t} \geq 5$ for $T / T_{c} \leq 1.5$. The fi nite-temperature transition is fi rst-order, and its strength increases with $N$. This allows us to consider spatial sizes $N_{s}=3 N_{t}$ only, and still maintain good control over the thermodynamic limit. We check this by varying $N_{s}$ in the potentially most problematic regime, for $S U(4)$ near $T_{c}$ : no measurable fi nite-size effect is found.

Like for $S U(2)$, we can compare our numerical results with perturbation theory, which is available to $\mathscr{O}\left(g^{3}\right)[2]$ :

$$
\frac{\tilde{\sigma}_{k}}{T^{2}}(T)=\frac{4 \pi^{2}}{3 \sqrt{3}} \times \frac{1}{\sqrt{\left(g^{2} N\right)(T)}} \times k(N-k) \times\left(1-15.27853 . . \frac{\left(g^{2} N\right)(T)}{16 \pi^{2}}\right)
$$

Systematic errors of any kind will tend to cancel in the ratio $\widetilde{\sigma}_{k} / \widetilde{\sigma}_{1}$. Our results are presented 


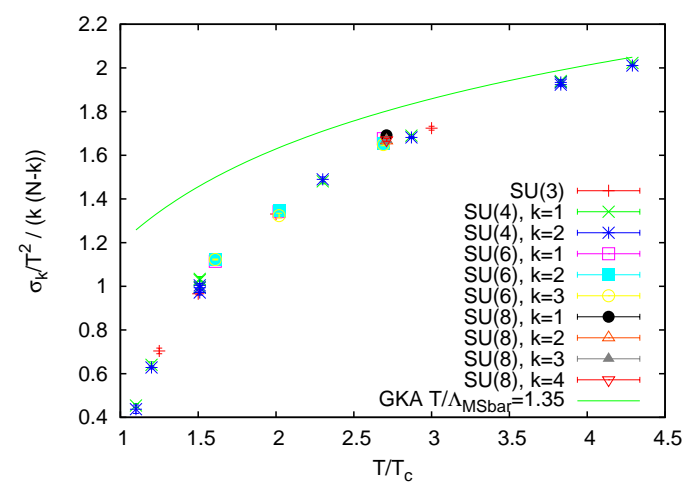

Figure 6: $\widetilde{\sigma}_{k} / T^{2}$, divided by the Casimir $k(N-$ $k)$, as a function of temperature. Data for all $S U(N), N \geq 3$ gauge groups and all values of $k$ collapse on a single curve, which deviates considerably from the perturbative prediction (solid curve) which worked so well for $\widetilde{\sigma}_{k} / \widetilde{\sigma}_{1}$ Fig. 5.

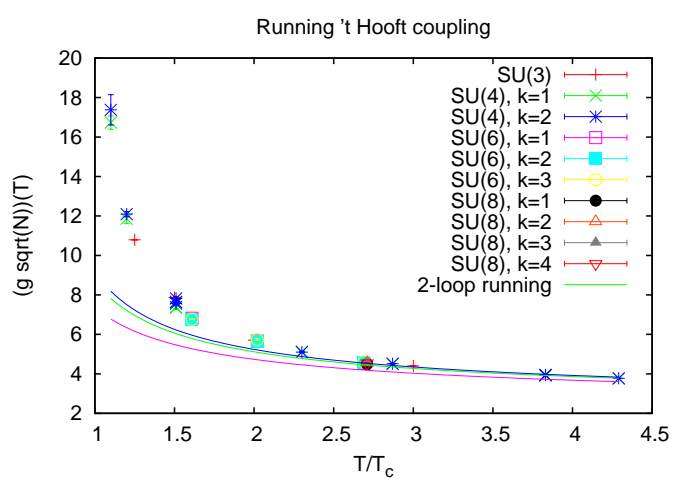

Figure 7: Running 't Hooft coupling $(\tilde{g} \sqrt{N})(T)$ extracted from $\widetilde{\sigma}_{k} / T^{2}$, as a function of temperature. The solid lines show 2-loop running, with $T_{c} / \Lambda_{\overline{M S}}=1.10,1.25$ and 1.35 .

in Fig. 5 as a function of $T / T_{c}$, for gauge groups $S U(4), S U(6)$ and $S U(8){ }^{1}$. The accuracy on $\widetilde{\sigma}_{k} / \widetilde{\sigma}_{1}$ is $1-3 \%$. In the $S U(4)$ case, a scaling test (from $N_{t}=5$ to 6 time-slices) shows no signifi cant scaling violations. The horizontal lines in Fig. 5 correspond to the leading and subleading order perturbation theory, i.e. the ratio of Casimirs $\frac{k(N-k)}{N-1}$. One can see excellent agreement with the data at high temperature, as expected, with only tiny downward deviations at lower temperatures $T \searrow T_{c}$.

Moreover, these tiny deviations are consistent with the bending curves in Fig. 5, which show the $\mathscr{O}\left(g^{3}\right)$ perturbative prediction [7]. The latter is expressed as a function of $T / \Lambda_{\overline{M S}}$, whereas in our simulations we fi x $T / T_{c}$. The needed factor $T_{c} / \Lambda_{\overline{M S}}$ is not known accurately, but a large variation between the accepted bounds 1.10 - 1.35 [9] in $S U(4)$ has almost no visible effect: the corresponding curves in Fig. 5(left) are almost indistinguishable. Thus, agreement of $\widetilde{\sigma}_{k} / \widetilde{\sigma}_{1}$ with $\mathscr{O}\left(g^{3}\right)$ perturbation theory persists in all our simulations at all temperatures studied. Disagreement, if it occurs, should be most visible for $S U(4)$, since this is the case where the deconfi nement transition is the weakest. This is also the most numerically diffi cult case, because the interface tension becomes quite small (thus harder to measure) as $T \searrow T_{c}$. Nevertheless, we observe consistency with perturbation theory, even for the smallest temperature where we can maintain suffi cient accuracy, namely $T / T_{c} \sim 1.1$.

Since agreement with perturbation theory is so good for $\widetilde{\sigma}_{k} / \widetilde{\sigma}_{1}$, one might expect the same for individual $k$-tensions $\widetilde{\sigma}_{k}$. This is not at all the case, as shown in Fig. 6. This fi gure shows $\frac{\widetilde{\sigma}_{k} / T^{2}}{k(N-k)}$, as a function of $T / T_{c}$, for all the gauge groups $S U(N), N>2$ and $k$-values we have considered. The solid line in the figure shows the $\mathscr{O}\left(g^{2}\right)$ perturbative prediction, which is independent of $N$ and $k$ (The $N$-, $k$-dependent $\mathscr{O}\left(g^{3}\right)$ correction is very small). Large deviations from perturbation theory are visible, showing that the interface tension sharply decreases near $T_{c}$, a phenomenon not captured by the perturbative expansion which is blind to the phase transition. Nevertheless, the departure from perturbation theory appears to be universal: data for all gauge groups collapse,

\footnotetext{
${ }^{1}$ The temperature is obtained from the determination of the $T=0$ string tension at the same coupling $\beta$ [8].
} 
even for $S U(3)$ (The $S U(2)$ data, which are not shown, lie signifi cantly below the rest). Thus, the $S U(N=\infty)$ limit is approached very fast, and one single curve $\frac{\widetilde{\sigma}_{k} / T^{2}}{k(N-k)}\left(\frac{T}{T_{c}}\right)$ appears suffi cient to give a good, non-perturbative description of all $S U(N), N>2$ interface tensions at all temperatures. This universal, non-perturbative dependence can be expressed via a running coupling constant, whose defi nition is taken to agree with the lowest order perturbative prediction:

$$
(\tilde{g} \sqrt{N})(T) \text { defi ned by } \frac{\tilde{\sigma}_{k}}{T^{2}}(T)=\frac{4 \pi^{2}}{3 \sqrt{3}} \times \frac{1}{\sqrt{(-2 / g)(T)}} \times k(N-k)
$$

This running coupling is shown in Fig. 6 as a function of $T / T_{c}$, together with the 2-loop running coupling, where the curves correspond to $T_{c} / \Lambda_{\overline{M S}}=1.10,1.25$ and 1.35. Agreement occurs for $T \gtrsim 3 T_{c}$. At lower temperatures, the coupling defi ned by the interface tension rises faster than perturbation theory would predict. A similar phenomenon can be seen in other quantities, e.g. the $S U(N)$ pressure [10]. Therefore, one might try to explain the two together, using the single coupling $(\tilde{g} \sqrt{N})(T)$ and low-order perturbation theory. This naive attempt fails quantitatively. Therefore, the pressure and the interface tension give us two independent properties of the $S U(N=\infty)$ theory, which are related by non-perturbative dynamics.

Note that Ref. [11] has performed a similar $S U(N)$ study to ours, but has measured instead derivatives of $\widetilde{\sigma}_{k}$ with respect to the lattice coupling, $d \widetilde{\sigma}_{k} / d \beta$. While their fi ndings for this quantity, i.e. Casimir scaling, agree with ours for the $\widetilde{\sigma}_{k}$ 's, the claim they make (Casimir scaling of the $\widetilde{\sigma}_{k}$ 's down to $T \sim 1.02 T_{c}$ ) is not substantiated by their data. Casimir scaling of the $\widetilde{\sigma}_{k}$ 's implies the same for the derivatives, but the converse is not true, because the integration constant (which dominates the value of $\widetilde{\sigma}_{k}$ near $T_{c}$ ) is not determined in [11]. Our fi ndings provide a justifi cation for their claim.

\section{References}

[1] P. de Forcrand, M. D’Elia and M. Pepe, Phys. Rev. Lett. 86 (2001) 1438 [arXiv:hep-lat/0007034].

[2] T. Bhattacharya, A. Gocksch, C. Korthals Altes and R. D. Pisarski, Nucl. Phys. B 383 (1992) 497 [arXiv:hep-ph/9205231].

[3] P. de Forcrand, B. Lucini and M. Vettorazzo, arXiv:hep-lat/0409148.

[4] P. de Forcrand and D. Noth, arXiv:hep-lat/0506005, to appear in Phys. Rev. D.

[5] S. z. Huang and M. Lissia, Nucl. Phys. B 438 (1995) 54 [arXiv:hep-ph/9411293].

[6] J. Fingberg, U. M. Heller and F. Karsch, Nucl. Phys. B 392 (1993) 493 [arXiv:hep-lat/9208012].

[7] P. Giovannangeli and C. P. Korthals Altes, Nucl. Phys. B 608 (2001) 203 [arXiv:hep-ph/0102022].

P. Giovannangeli and C. P. Korthals Altes, Nucl. Phys. B 721 (2005) 1 [arXiv:hep-ph/0212298].

P. Giovannangeli and C. P. Korthals Altes, Nucl. Phys. B 721 (2005) 25 [arXiv:hep-ph/0412322].

[8] B. Lucini, M. Teper and U. Wenger, JHEP 0406 (2004) 012 [arXiv:hep-lat/0404008].

[9] M. Laine and Y. Schröder, JHEP 0503 (2005) 067 [arXiv:hep-ph/0503061].

[10] K. Kajantie, M. Laine, K. Rummukainen and Y. Schröder, Phys. Rev. D 67 (2003) 105008 [arXiv:hep-ph/0211321]; B. Bringoltz and M. Teper, arXiv:hep-lat/0506034.

[11] F. Bursa and M. Teper, JHEP 0508 (2005) 060 [arXiv:hep-lat/0505025]. 